Document downloaded from:

http://hdl.handle.net/10251/145986

This paper must be cited as:

Simarro, MA.; Puig, B.; Martínez Zaldívar, FJ.; Gonzalez, A. (10-2). Combined precoding for multiuser Multiple-Input Multiple-Output satellite communications. Computers \& Electrical Engineering. 71:704-713. https://doi.org/10.1016/j.compeleceng.2018.08.006

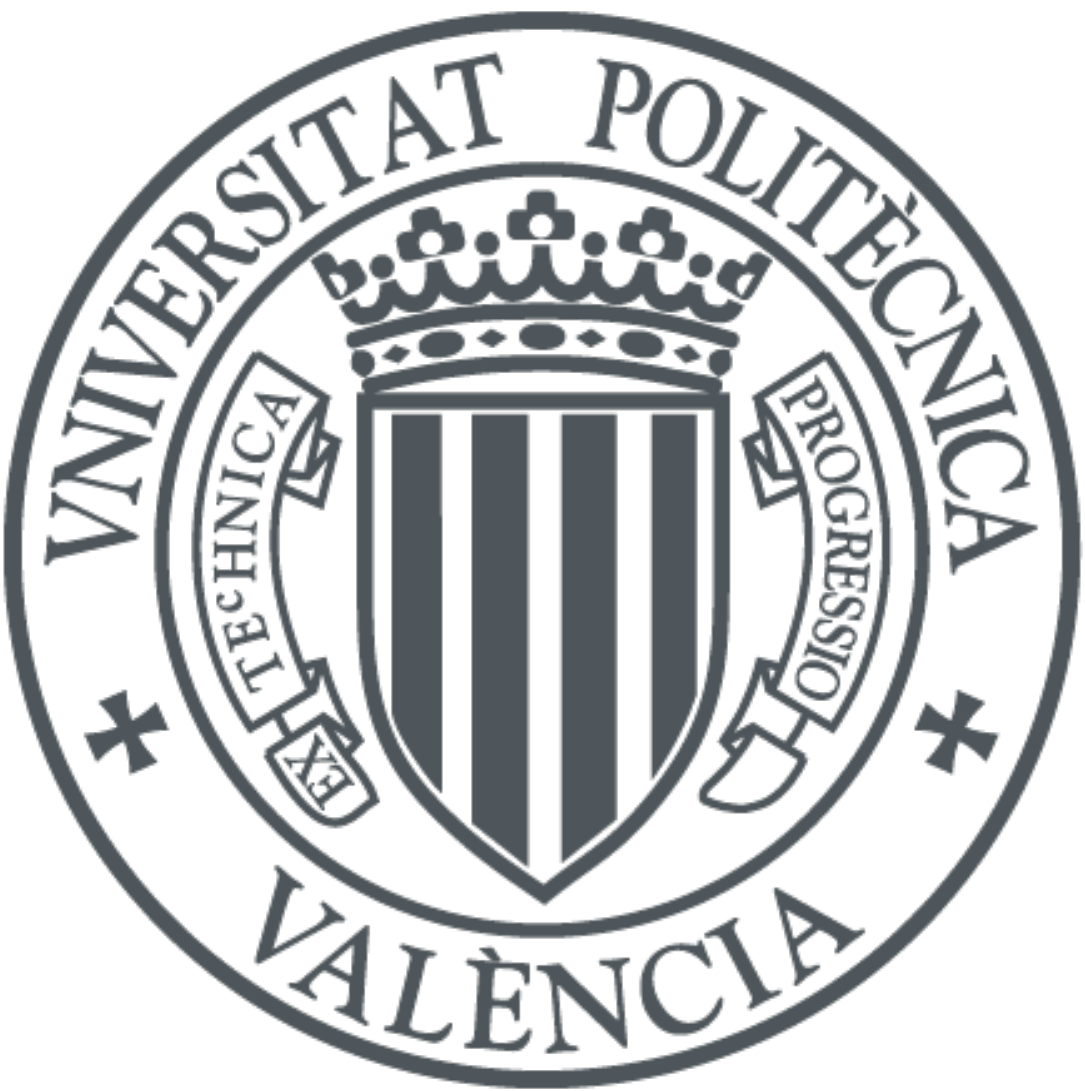

The final publication is available at

https://doi.org/10.1016/j.compeleceng.2018.08.006

Copyright Elsevier

Additional Information 


\title{
Combined Precoding for Multiuser Multiple-Input Multiple-Output Satellite Communications
}

\author{
M. Ángeles Simarro*, Beatriz Puig, F.J. Martínez-Zaldívar, Alberto Gonzalez \\ Institute of Telecommunications and Multimedia Applications, Universitat Politècnica de \\ València, Camino de Vera s/n 46022 Valencia, SPAIN.
}

\begin{abstract}
Applying Multiple-Input Multiple-Output (MIMO) techniques in satellite communications can increase data rates. However, new signal processing elements have to be taken into account to fully exploit the expected advantages of MIMO communications. In this paper, we evaluate different precoding techniques over the satellite channel. A performance comparison between several precoders in terms of Bit Error Rate (BER) and complexity is given for different channel realizations. Furthermore, a novel hybrid scheme for signal precoding is proposed that optimizes the computation for a required BER. The new scheme is based on the matrix condition number of the satellite MIMO channel.

Keywords: Precoding, MIMO, Satellite communications, Complexity, Matrix condition number
\end{abstract}

\section{INTRODUCTION}

The potential of Multiple-Input Multiple-Output (MIMO) techniques in terrestrial wireless networks has been demonstrated during the last decades [1. MIMO systems can achieve higher data rates compared to Single-Input SingleOutput (SISO) systems because the channel capacity can be increased linearly with the number of transmit and receive antennas.

\footnotetext{
* Corresponding author

Email addresses: mdesiha@iteam.upv.es (M. Ángeles Simarro), beapuila@teleco.upv.es (Beatriz Puig), fjmartin@dcom.upv.es (F.J. Martínez-Zaldívar), agonzal@dcom.upv.es (Alberto Gonzalez)
}

Preprint submitted to Journal of ${ }^{A} T_{E} X$ Templates

July 23, 2018 
For this reason, a great interest has been generated due to its possible application to satellite networks in order to reach higher data rates [2. Nevertheless, there exist elementary differences between terrestrial and satellite channels, which prevent the direct application of MIMO techniques to satellite communications. Therefore, it can be considered a hot research topic. These differences are mainly related to the channel, especially with the Line-Of-Sight (LOS) operation of satellite links and to the absence of scattering effects in the proximity of the satellite, which eliminates multipath fading profiles over the space segment and leads to an inherent rank deficiency of the MIMO channel matrix [3].

Many techniques, mainly developed for terrestrial systems, and applied at either the receiver or the transmitter or both, can reduce or almost eliminate the impact of the unavoidable inter-user interference [4. In order to mitigate the interferences and allow cost and complexity reductions of the user terminal, precoding techniques are also attractive for mobile satellite transmission schemes. Recent research has explored the use of precoding techniques in single beam systems and in multi-beam systems [5] [6]. However, the high number of transmit antenna elements and users limit the precoding designs due to optimization complexity.

A number of linear and non-linear techniques have been recently proposed to effectively manage interference among users. Between them, Dirty Paper Coding (DPC) 7] is an optimum precoding technique that allows the cancellation of the interference without power penalty. However, its high complexity restricts its implementation in practical systems, thus suboptimal techniques have to be used instead. In the literature of satellite communications, most of the recent work focuses on the conventional scenario where polarization or partial frequency reuse is employed to mitigate inter-beam interference [6] 8$]$.

An analysis of some of the most commonly used precoding techniques in wireless communications has been conducted in previous works 9 [10]. Nevertheless, the performance of each precoding scheme in multiuser downlink satellite communications has not been considered yet. For this reason, the present work deals with the performance of these precoders for multiuser downlink satellite 
communications, paying special attention to their performance in terms of Bit Error Rate (BER) and computational complexity.

On the other hand, previous works have shown that the performance of MIMO detectors is highly influenced by the MIMO channel matrix condition number [11. This behavior has been analyzed for the precoding methods under a multiuser downlink satellite communications. As a result of this study and in order to improve the precoding efficiency a hybrid precoding scheme is proposed. This scheme aims to reduce the computational cost at the transmitter without having a high penalty in the quality of the received signal.

Thus a combining scheme based on the channel matrix condition number is proposed. This scheme selects the precoding algorithm applied to the transmitted signal based on the condition number of the channel matrix following a similar strategy to the combined decoder proposed in [11. The main idea of this method is to minimize the impact of the quality of channel on the detector performance. The proposed hybrid scheme reduces the computational cost with minimum BER performance degradation.

In summary, the main contributions of this paper are the followings: an evaluation of the performance of several precoders schemes under a multiuser downlink satellite communication system, a study of the influence of the channel matrix condition number in the performance of this precoders and the proposal of a new precoding scheme.

The rest of the paper is organized as follows: In Section 2 the system and satellite channel model are described. Section ?? illustrates MIMO precoding algorithm functioning. The BER and complexity performances of the previous detectors are exposed in Section 4. The novel hybrid scheme is introduced in Section 5 and its performance analyzed. Finally, several conclusions are drawn in Section 6 . 


\section{SYSTEM AND CHANNEL MODEL}

\subsection{SYSTEM MODEL}

We consider the downlink of a land-mobile receiving diversity satellite system consisting of a $P$ dual polarized satellites (and therefore, $N=2 P$ antennas) and $M$ mobile receiving users with a single non-polarized antenna [12. The system model can be represented as

$$
\mathbf{y}^{c}=\mathbf{H}^{c} \mathbf{x}^{c}+\mathbf{n}^{c},
$$

where $\mathbf{y}^{c}$ denotes the complex received $M \times 1$ vector. The channel matrix $\mathbf{H}^{c}$ is formed by $M \times N$ complex elements and each component $h_{i j}^{c}$ represents the channel between the $j$-th transmit antenna and the $i$-th user. Vector $\mathbf{x}^{c}$ represents the transmitted signal that depends on the precoding algorithm and it is built from the vector $\mathbf{s}^{c}$, which is the $M \times 1$ vector of data symbols. In this paper, $\mathbf{s}^{c}$ vector contains the complex symbols chosen from a $\beta$-QAM constellation. Vector $\mathbf{n}^{c}$ is a Gaussian noise vector with zero mean and certain variance.

\subsubsection{SATELLITE CHANNEL MODEL}

The Loo distribution is often used for the modeling of Land-Mobile Satellite (LMS) channels, for instance in DVB-SH (Digital Video Broadcasting Satellite services to Handhelds). In this model, the complex fading gain from the $j$-th transmit antenna to the $i$-th user can be represented by a summation of two parts as [13]-15]:

$$
h_{i j}^{c}=\sqrt{\frac{K}{1+K}} h_{i j}^{c}{ }^{(L O S)}+\sqrt{\frac{1}{1+K}} h_{i j}^{c}{ }^{(N L O S)},
$$

where $h_{i j}^{c}{ }^{(L O S)}$ models the shadowing effect of the channel and its entries are generated using the Log-normal distribution with parameters $\alpha$ (mean) and $\psi$ (standard deviation), $h_{i j}^{c}(N L O S)$ is the multipath component of the channel with Rayleigh distributed entries with parameter $M P$ (the average power of the Rayleigh distributed envelope of the small-scale fading components) and 
$K$ is the Rician $K$-factor (alternatively described by the multipath power $M P$ corresponding to the coefficient of $h_{i j}^{c}(N L O S)$ ). When the value of $K$ is large, a LOS path is considered. Nevertheless, if $K$ value is small, a scattered path is taken into account. Typical values of these parameters can be found in [15]. The equivalent real-valued representation of (1) is denoted as $\mathbf{y}=\mathbf{H x}+\mathbf{n}$, by setting

$$
\mathbf{y}=\left[\begin{array}{l}
\Re\left(\mathbf{y}^{c}\right) \\
\Im\left(\mathbf{y}^{c}\right)
\end{array}\right], \quad \mathbf{x}=\left[\begin{array}{l}
\Re\left(\mathbf{x}^{c}\right) \\
\Im\left(\mathbf{x}^{c}\right)
\end{array}\right], \quad \mathbf{n}=\left[\begin{array}{l}
\Re\left(\mathbf{n}^{c}\right) \\
\Im\left(\mathbf{n}^{c}\right)
\end{array}\right]
$$

and

$$
\mathbf{H}=\left[\begin{array}{ll}
\Re\left(\mathbf{H}^{c}\right) & -\Im\left(\mathbf{H}^{c}\right) \\
\Im\left(\mathbf{H}^{c}\right) &
\end{array}\right] .
$$

This real representation will be considered throughout the rest of the paper.

\section{PRECODING TECHNIQUES}

In a broadcast MIMO system with multiple users such as the one studied in this paper, the physical distance between users prevents joint processing at reception. Therefore, since multiuser MIMO downlink interference is known at the transmitter, a precoder can be used to annihilate this effect.

Precoding techniques can mitigate the multiuser interference in MIMO systems [16, even when the users are equipped with a single antenna, so the complexity of the receiver can be significantly simplified. The most employed precoding techniques are briefly described throughout this section. It is important to note that precoding is carried out assuming perfect knowledge of Channel State Information (CSIT) at the transmitter.

The precoders evaluated in this paper are based on the Vector Perturbation (VP) technique. Thus, the precoded signal can be expressed as

$$
\mathbf{x}=\mathbf{H}^{\dagger}(\mathbf{s}+\mathbf{p})
$$

where $\mathbf{s}$ is the equivalent real vector containing the original data symbols for each user, $\mathbf{p}$ is the perturbation vector and $\mathbf{H}^{\dagger}$ is the pseudoinverse channel matrix. 
The optimal perturbation vector $\mathbf{p}$ minimizes the power of the transmitted signal. In order to allow the users to remove the perturbation, $\mathbf{p}$ is set as

$$
\mathbf{p}=\tau \boldsymbol{\Lambda}
$$

where $\tau$ is a positive real number and $\boldsymbol{\Lambda}$ is a $2 M$-dimensional vector which depends on $\mathbf{H}^{\dagger}$ and the precoding method. The value of $\tau$ is chosen such that the point from the symbol constellation can be uniquely recovered. A possible value of $\tau$ for a $\beta$-QAM constellation is given by $\tau=2 \sqrt{\beta}$. Detailed discussion about the value of $\tau$ can be found in [17. At the receivers, users remove the perturbation by applying

$$
y_{m} \quad \bmod \quad \tau:=y_{m}-\tau\left\lfloor\frac{y_{m}+\tau / 2}{\tau}\right\rfloor
$$

being $m=1,2, \ldots, 2 M . y_{m}$ denotes the received signal at each user and $\lfloor\cdot\rfloor$ operator maps a real number to the lower integer.

\subsection{ZERO-FORCING PRECODER}

Zero-Forcing (ZF) precoding [18] consists in applying the channel inversion at the transmitter. It can be considered as a particular case of VP where $\mathbf{p}=\mathbf{0}$, so the modulus operation is not required at the receiver. The inversion matrix can be computed as the Moore-Penrose pseudoinverse of the channel matrix, thus the precoded signal can be expressed as:

$$
\mathbf{x}=\mathbf{H}^{\dagger} \mathbf{s}=\mathbf{H}^{H}\left(\mathbf{H H}^{H}\right)^{-1} \mathbf{s}
$$

\subsection{TOMLINSON-HARASHIMA PRECODER}

Tomlinson-Harashima Precoder (THP) is equivalent to VP where the perturbation vector $\mathbf{p}$ is obtained sequentially and efficiently through feedback filtering and modulus $\tau$ operation. The modulus operator reduces the power of the transmitted signal compared to a linear precoding scheme [16]. In the THP scheme, the channel matrix is decomposed as a $\mathbf{H}=\mathbf{L}_{0} \mathbf{Q}_{0}$ and the matrices that take part in the precoding algorithm are computed by 


$$
\begin{gathered}
\mathbf{L}=\mathbf{L}_{0} \mathbf{G}^{-1} \\
\mathbf{Q}=\mathbf{G Q}_{0},
\end{gathered}
$$

where $\mathbf{L} \in \mathbb{R}^{2 M \times 2 M}$ is a lower unit triangular matrix, $\mathbf{Q} \in \mathbb{R}^{2 M \times 2 N}$ has orthogonal rows and $\mathbf{G}$ is a diagonal matrix containing the diagonal of $\mathbf{L}_{0}$.

The precoded symbols can be initially expressed as

$$
\begin{gathered}
\hat{x}_{m}=s_{m}-\sum_{t=1}^{m-1} l_{m, t} \tilde{x}_{t}, \\
\tilde{x}_{m}=\hat{x}_{m} \bmod \tau,
\end{gathered}
$$

with $m=1, \ldots, 2 M$. Modulus operation is applied in order to restrict the symbols to the original constellation boundaries. Finally, the transmitted signal over the channel is computed as $\mathbf{x}=\mathbf{Q}^{\dagger} \tilde{\mathbf{x}}=\mathbf{Q}_{0}^{H} \mathbf{G}^{-1} \tilde{\mathbf{x}}$.

\subsection{LATTICE-REDUCTION-AIDED PRECODING}

Lattice-Reduction-Aided Precoding (LRAP) makes use of Lattice-Reduction (LR) techniques [19]20] to obtain an efficient approximation of the optimal perturbation given by (6). The LR techniques consist of finding another base with better orthogonality properties than the original one. Different reduction techniques have been proposed, however lattice reduction method proposed by Lenstra, Lenstra and Lovász (LLL algorithm) 21] is the most employed one because it offers a good trade-off between performance and complexity. The transformation is performed on a $\mathbf{B}$ matrix, so that $\mathbf{B}=\tilde{\mathbf{B}} \mathbf{T}$, where $\mathbf{T}$ is the unimodular transformation matrix with integer elements, $\mathbf{B}$ depends on the precoding technique and $\tilde{\mathbf{B}}$ is the lattice-reduced matrix.

In 22] two different LRAP techniques were presented; the LRAP Linear and the LRAP VB (V-BLAST, Vertical Bell Laboratories layered space-time). Moreover, 23] proposes a new scheme, the LRAP THP, where THP is applied after performing a LR technique over the channel matrix. 
- LRAP Linear.

In this scheme, the LR stage is applied to the columns of the psudeoinverse matrix $\mathbf{H}^{\dagger}$, giving

$$
\mathbf{H}^{\dagger}=\tilde{\mathbf{H}}^{\dagger} \mathbf{T}
$$

where $\tilde{\mathbf{H}}^{\dagger}$ is the lattice-reduced channel matrix.

Then, the perturbation vector is computed using the rounding off approximation as

$$
\mathbf{p}=-\tau \mathbf{T}^{-1}\left\lceil\frac{\mathbf{T s}}{\tau}\right\rfloor,
$$

where $\lceil\cdot\rfloor$ operator maps a real number to the nearest integer.

- LRAP VB.

The LRAP VB is another variant of this algorithm which is based on Successive Interference Cancellation (SIC), where the VBLAST algorithm is applied [22]. In this precoder the matrices $\mathbf{Q}$ and $\mathbf{L}$ matrix can be simply calculated from a QR-type decomposition of $\tilde{\mathbf{H}}^{\dagger}$ such that

$$
\mathbf{Q} \tilde{\mathbf{H}}^{\dagger}=\mathbf{L}
$$

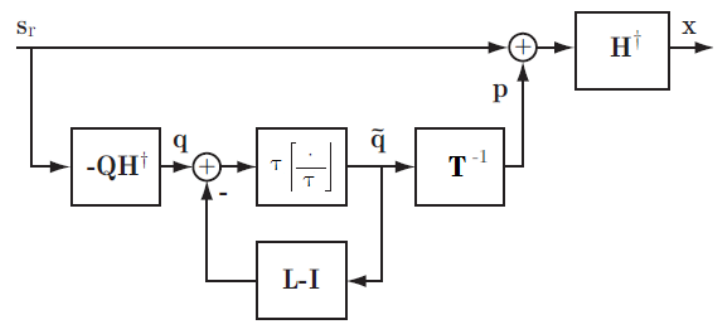

Figure 1: Lattice-Reduced-Aided Precoder VB Scheme.

As we can see in the Fig. 1 the first step of the algorithm computes the vector

$$
\mathbf{q}=-\mathbf{Q H}^{\dagger} \mathbf{s}
$$


Then, the components of $\tilde{\mathbf{q}}$ are calculated as

$$
\tilde{q}_{m}=\tau\left\lceil\frac{q_{m}-\sum_{l=1}^{m-1} L_{m, l} \tilde{q}_{l}}{\tau}\right\rfloor \quad m=1, \ldots, 2 M .
$$

Last of all, the perturbation vector $\mathbf{p}$ in Fig. 1 can be expressed as

$$
\mathbf{p}=\mathbf{T}^{-1} \tilde{\mathbf{q}}
$$

- LRAP THP.

In 23] a new scheme is presented, where THP is applied after performing an LR technique over the channel matrix. In this scheme the vector $\mathbf{s}$ is replaced by the new vector $\tilde{\mathbf{s}}=\mathbf{T}^{-1} \mathbf{s}$ and the QL decomposition given by 9 is performed over the lattice-reduced channel matrix.

\section{PERFORMANCE AND COMPLEXITY}

In this section, performance and complexity comparisons among the precoding algorithms explained in the previous section have been carried out. A 4-QAM constellation has been used in all the simulations, regarding the number of transmit antennas and the number of users, a $4 \times 4$ system scenario has

been considered, which consists of two dual-polarized satellites and 4 mobile users equipped with a non-polarized antenna.

\subsection{PERFORMANCE FOR SATELLITE CHANNEL}

In this section, a performance comparison is given among the precoded communications over the satellite channel. Several parameters of the Loo distribution have been modified to analyze their influence in the performance in terms of BER. The Rician factor $K$ in (2) represents the power distribution for each part of the channel, high values mean that the LOS path predominates, however when the Rician factor gets lower the channel becomes more scattered. It has been proved, by fixing the others channel parameters, that variations on the Rician factor only produces a displacement on the BER performance 
graph. Therefore, for simplicity this parameter has been set to $0 \mathrm{~dB}$ in all the simulations.

The distribution's parameters chosen for comparison have been extracted from [15] for urban area and different levels of shadow and elevation angles. Two levels of shadow and two different elevation angles have been selected to compare the precoders performance under extreme conditions.

First, an elevation angle of $40^{\circ}$ has been chosen for two levels of shadow. Fig. $2 \mathrm{a}$ represents the BER performance for a line-of-sight case and Fig. $2 \mathrm{~b}$ for a deep shadow case. We can see how as the level of shadow increases, the BER performance worsens. Further, it is important to note that the LR based algorithms achieve better performance than the ZF and THP algorithms for both cases.

The same analysis has been carried out for an angle elevation of $10^{\circ}$ and the two different states of shadowing. Fig. 2c illustrates how the LRAP precoders continue showing the best performance. However, Fig. 2d shows that the LRAP THP is the only precoder that gets a practical performance for the worst channel, angle elevation of $10^{\circ}$ and deep shadow. In conclusion, the LRAP THP outperforms all the other methods for all satellite channel conditions.

\subsection{COMPLEXITY}

Notwithstanding, it is interesting to compare the arithmetic complexity of the algorithms. In [9] the computational cost among the precoding techniques considered in this paper was carried out. It was proved that the overall cost depends on the duration of the interval where the channel remains unchanged ( $L_{c h}$ represents the number of vector symbols transmitted through the same channel, being unnecessary to renew the knowledge of the channel). As detailed in [9], Tables 1 13 have to be taken into account in order to compute the total number of arithmetic operations.

There are some differences between the complexity presented in [9] and the complexity evaluated here. For the ZF implementation, the THP scheme without the modulus operation has been considered because the $\mathrm{QR}$ decomposition 


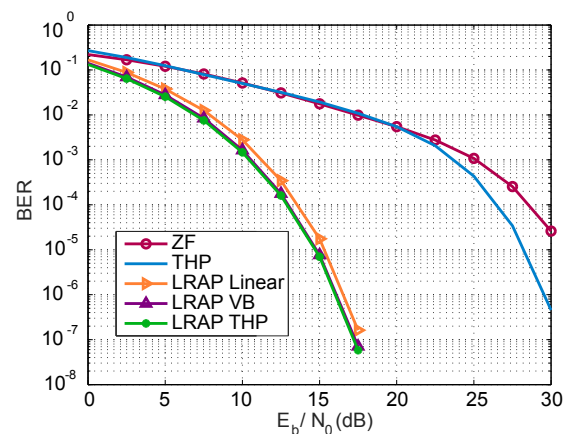

(a) Line-of-sight and elevation angle $40^{\circ}$ $(\alpha=-0.2 \mathrm{~dB}, \psi=1 \mathrm{~dB}, M P=-32.9 \mathrm{~dB})$

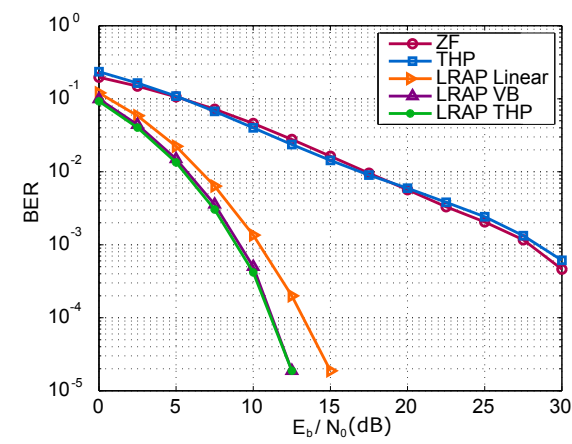

(c) Line-of-sight and elevation angle $10^{\circ}$ $(\alpha=-0.7 \mathrm{~dB}, \psi=1.9 \mathrm{~dB}, M P=-38.3 \mathrm{~dB})$

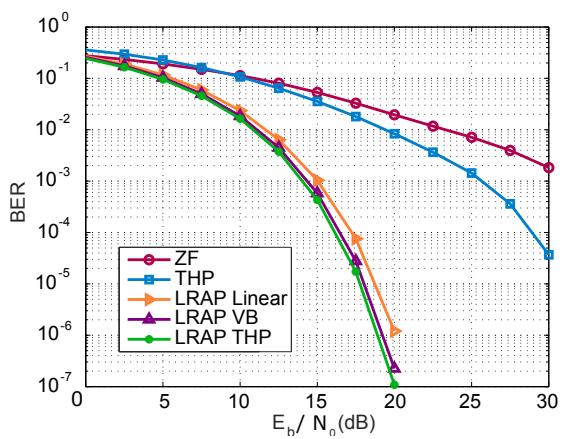

(b) Deep shadow and elevation angle $40^{\circ}$ $(\alpha=-15.1 \mathrm{~dB}, \psi=2.6 \mathrm{~dB}, M P=-16 \mathrm{~dB})$

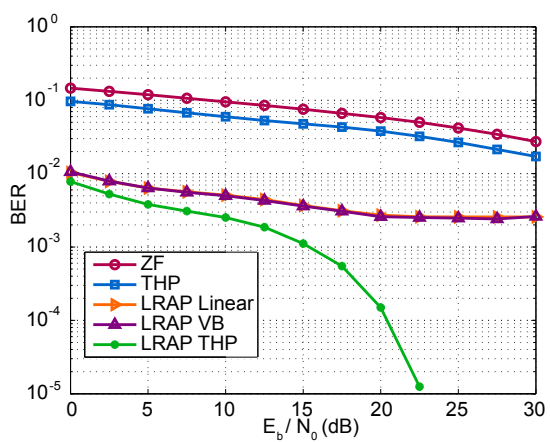

(d) Deep shadow and elevation angle $10^{\circ}$

Figure 2: BER performance for a $4 \times 4$ MIMO satellite channel using several precoders with $K=0 \mathrm{~dB}$.

has less computation complexity than the pseudoinverse operation.

It can also be noted that in Table 2 the $\mathbf{Q}^{\dagger}$ operation has not been considered for THP and LRAP THP schemes since this pseudoinverse can be performed as $\mathbf{Q}^{\dagger}=\mathbf{Q}_{0}^{H} \mathbf{G}^{-1}$.

It is important to note that the complexity cost depends only on the dimension of the system, not on the modulation order, as the Tables $1+3$ show. The total number of arithmetic operations for the different precoding algorithms has 


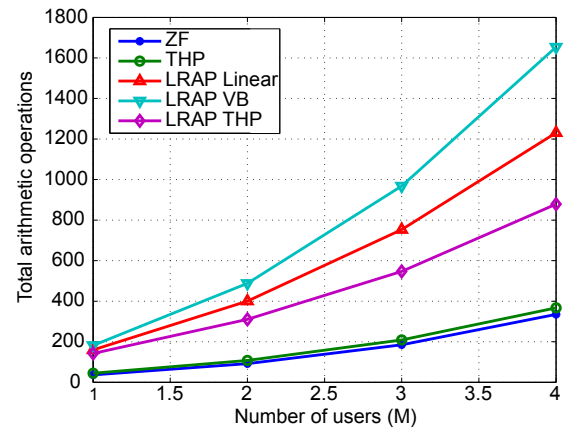

(a) $L_{c h}=5$

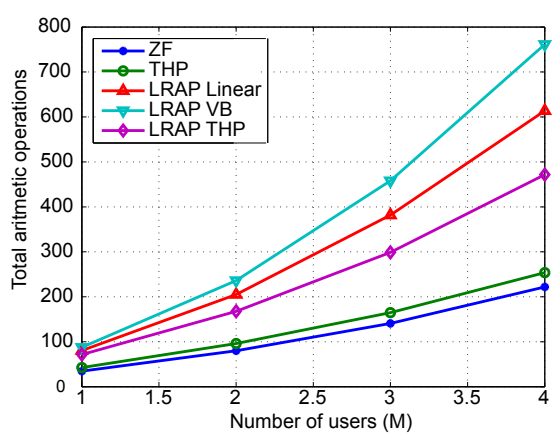

(b) $L_{c h}=20$

Figure 3: Total number of arithmetic operations of precoding algorithms for a system with $N=4$.

\begin{tabular}{c|c|c} 
& Sums & Products \\
\hline ZF & $2 M^{2}+4 M N-M-2 N$ & $2 M^{2}+4 M N-M+2 N$ \\
\hline THP & $2 M^{2}+4 M N+3 M-2 N$ & $2 M^{2}+4 M N+3 M+2 N$ \\
\hline LRAP Linear & $8 M^{2}+4 M N+2 M-2 N$ & $8 M^{2}+4 M N+4 M+2 N$ \\
\hline LRAP VB & $10 M^{2}+4 M N+M-2 N$ & $10 M^{2}+4 M N+3 M+2 N$ \\
\hline LRAP THP & $6 M^{2}+4 M N+M-2 N$ & $6 M^{2}+4 M N+3 M+2 N$ \\
\hline
\end{tabular}

Table 1: Per-symbol-vector arithmetic cost of precoding algorithms.

\begin{tabular}{c|c|c|c|c} 
& QR & LLL & $\mathbf{T}^{-1}$ & $\left(\mathbf{H H}^{H}\right)^{-1}$ \\
\hline ZF & Yes & No & No & No \\
\hline THP & Yes & No & No & No \\
\hline LRAP Linear & No & Yes & Yes & Yes \\
\hline LRAP VB & Yes & Yes & Yes & Yes \\
\hline LRAP THP & Yes & Yes & Yes & No \\
\hline
\end{tabular}

Table 2: Main preprocessing stages of precoding algorithms.

been evaluated for a system of $N=4$ and the number of users ranging from 1 to 4. Figs. $3 \mathrm{a}$ and $3 \mathrm{~b}$ show the cost for $L_{c h}=5$ and $L_{c h}=20$. As it can be seen in these figures, the LRAP Linear and LRAP VB precoders are the most 


\begin{tabular}{c|c|c} 
& Sums & Products \\
\hline ZF & - & $4 M N+2 M$ \\
\hline THP & - & $4 M N+2 M$ \\
\hline LRAP Linear & $16 M^{2} N-4 M^{2}-4 M N$ & $16 M^{2} N$ \\
\hline LRAP VB & $24 M^{2} N-8 M^{2}-4 M N$ & $24 M^{2} N$ \\
\hline LRAP THP & - & $4 M N+2 M$ \\
\hline
\end{tabular}

Table 3: Additional preprocessing arithmetic cost of precoding algorithms.

complex ones. However, the LRAP THP, which exhibits the best performance, requires less computation than the other LRAP algorithms. On the other hand, the ZF and THP are computationally less expensive.

It is important to analyze the different precoding schemes from a computational and performance point of view. The complexity or efficiency restrictions can help us to decide the most suitable precoder for a practical situation.

\section{HYBRID PRECODING}

As we have seen in previous sections, the algorithms that exhibit better performance against the interference between users are the most complex ones. There is interest in improving the quality of the received signal keeping a moderate computational cost and thus lightening the burden on the satellite on-board processors. Therefore, this scheme takes advantage of the impact of the channel matrix condition number in data precoding in order to decrease the complexity of already proposed precoding schemes.

\subsection{CONDITION NUMBER}

The matrix condition number is the ratio of the largest to the smallest singular values. The sensitivity of the solution of a non-singular system of linear equations $\mathbf{A x}=\mathbf{b}$ with respect to perturbations of the matrix $\mathbf{A}$ is directly related to this parameter [11] 24, which can be computed as $\mathcal{K}(\mathbf{A})=$ $\|\mathbf{A}\|\left\|\mathbf{A}^{-1}\right\|$. This means the condition number is a measure of the effect of 
errors when inverting A. All precoders calculate a pseudoinverse at some point, so the condition number will give us a hint about the conditioning of the matrix and its impact on the BER.

A channel matrix with a low condition number is said to be well-conditioned, which means its inverse could be computed with reasonable accuracy. However, a matrix with a high condition number is said to be ill-conditioned and its inverse is prone to large numerical errors.

MIMO channel matrix condition number affects precoders performance. An empirical study of the condition number for the typical satellite channels parameter extracted from [15 has been done. Fig. 4 shows for the case with $K=0 \mathrm{~dB}$, deep shadow and elevation angle $10^{\circ}(\alpha=-24.4 \mathrm{~dB}, \psi=9.4 \mathrm{~dB}$, $M P=-23.9 \mathrm{~dB}$ ) how BER performance increases as the condition number does. This Figure represents the results for the THP algorithm, however the other precoders give very similar results. Furthermore, it has been observed that the condition number worsens with increasing shadow and lower elevation angle. This way, when the satellite works under these circumstances, the matrix condition number generally increases and the communication system exhibits lower performance.

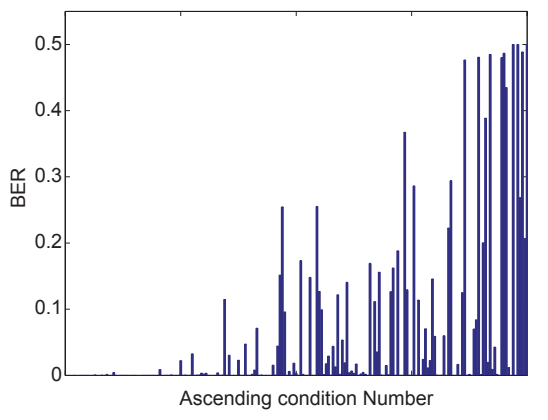

Figure 4: BER value as the condition number increases using THP for a $E_{b} / N_{0}=10 \mathrm{~dB}$ and a $4 \times 4$ MIMO system with $K=0 \mathrm{~dB}$, deep shadow and elevation angle $10^{\circ}(\alpha=-24.4 \mathrm{~dB}$, $\psi=9.4 \mathrm{~dB}, M P=-23.9 \mathrm{~dB})$. 


\subsection{HYBRID SCHEME}

This section exposes the operation of the proposed hybrid scheme based on the election of the algorithm used, which depends on the condition number of the channel realization. As previously explained, higher channel condition numbers mean that the channel is ill-conditioned and consequently, the BER increases. Thus, bad channel condition numbers need the use of a precoder with better performance at the expense of higher computational cost. However, when the condition number is lower than the threshold (well conditioned channel) a simpler precoder is selected. (see Fig. 5).

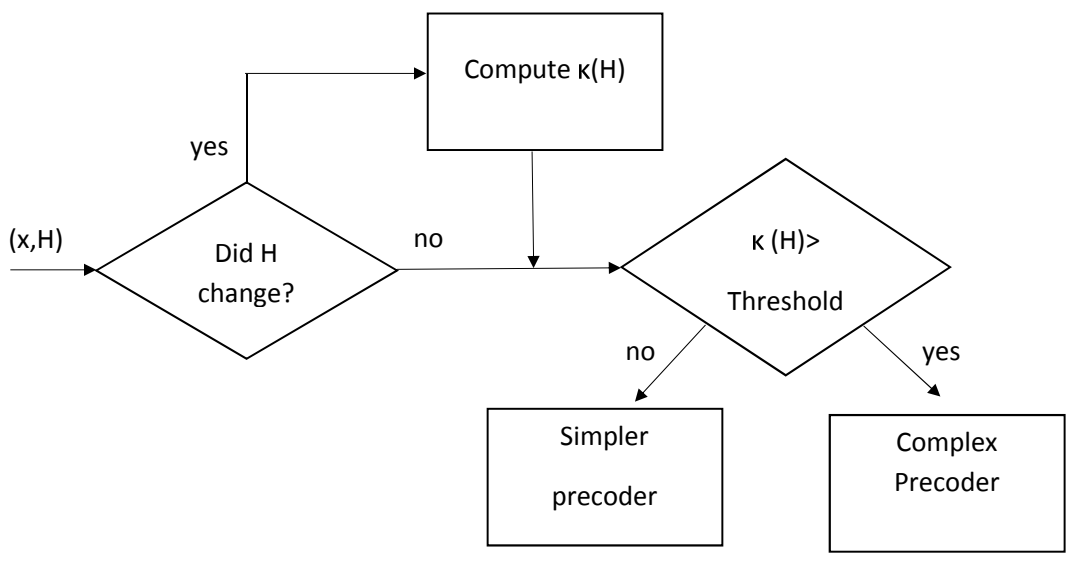

Figure 5: Flow diagram of the proposed hybrid scheme.

The best algorithms in terms of BER are the LRAP VB and the LRAP THP, which exhibit almost the same performance in all cases. Since the complexity of the LRAP THP is lower than the complexity of the LRAP VB, the chosen costly algorithm is the LRAP THP.

Therefore, the scheme proposed carries out a precoding algorithm that changes depending on the channel condition number. Therefore, when a poor condition number is detected, which is above a selected threshold, the precoder LRAP THP with better performance and more complexity is chosen. When the condition number is lower than the threshold (well-conditioned channel), it switches to a simpler precoder. In our simulations, the threshold was empirically selected, 
taking into account the usual values of the condition number. Furthermore, a low complexity method to estimate the condition number can be used [25].

Table 4 illustrates the percentage of computational cost reduction depending on the use of the simpler ZF and THP methods versus LRAP THP precoders for a $4 \times 4$ MIMO system. There, it can be seen that as the usage of the simpler precoder increases, the reduction of the computational cost is higher. Table 4 shows the reduction for $L_{c h}=5$, however similar results are obtained for a value of $L_{c h}=20$.

\begin{tabular}{|c|c|c|c|c|c|c|}
\hline \multirow{2}{*}{ Combination } & \multicolumn{5}{|c|}{ Percentage of use (Simpler Precoder vs LRAP THP) } \\
\cline { 2 - 7 } & $40 \%-60 \%$ & $50 \%-50 \%$ & $60 \%-40 \%$ & $70 \%-30 \%$ & $80 \%-20 \%$ & $90 \%-10 \%$ \\
\hline $\begin{array}{c}\text { ZF and } \\
\text { LRAP THP }\end{array}$ & $24.75 \%$ & $30.94 \%$ & $37.13 \%$ & $43.31 \%$ & $49.5 \%$ & $55.68 \%$ \\
\hline $\begin{array}{c}\text { THP and } \\
\text { LRAP THP }\end{array}$ & $23.29 \%$ & $29.12 \%$ & $34.94 \%$ & $40.76 \%$ & $46.58 \%$ & $52.41 \%$ \\
\hline
\end{tabular}

Table 4: Cost reduction percentage for $L_{c h}=5$ for a $4 \times 4$ MIMO system.

The combination with a simple precoder (two cases: ZF and THP) was done to obtain the maximum reduction of cost. The performance for the two combinations and different channel parameters and percentage of use has been evaluated. Figs. 6a to $7 \mathrm{~b}$ represent the performance results in terms of BER for different cases.

It is important to note the following results. In Fig. 6a the performance of the worst channel (deep shadow and elevation angle of $10^{\circ}$ ) has been evaluated for ZF, LRAP THP and the hybrid scheme with the combination of ZF (40\%) and LRAP THP (60\%). In this case, the hybrid's performance is almost identical to the LRAP THP one. Thus, a cost reduction of $24.75 \%$ is reached. Fig. 6b represents the results for the same channel and the combination of THP (40\%) and LRAP THP (60\%), in this case the hybrid scheme achieves the same BER performance than the LRAP THP with a $23.29 \%$ of cost reduction.

Figs. $7 \mathrm{a}$ and $7 \mathrm{~b}$ represent the results for a channel with line-of-sight and elevation angle of $40^{\circ}$. The simplest precoder is used during $70 \%$ of time achieving a 
cost reduction of $43.31 \%$ and $40.76 \%$ for the ZF and THP respectively. In these cases, the performance degradation is not significant and the hybrid scheme performance is close to the LRAP THP one.

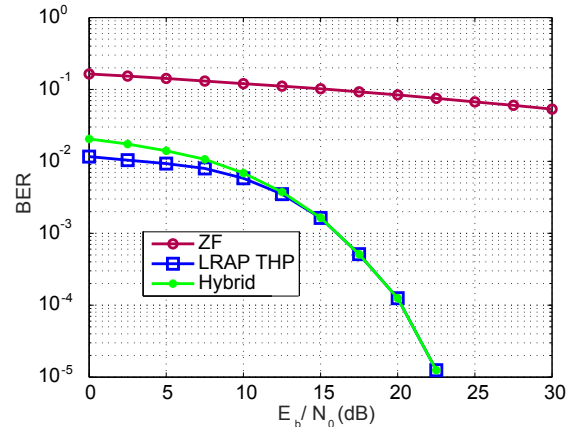

(a) Hybrid precoding with combination of $\mathrm{ZF}$ (40\%) and LRAP THP (60\%)

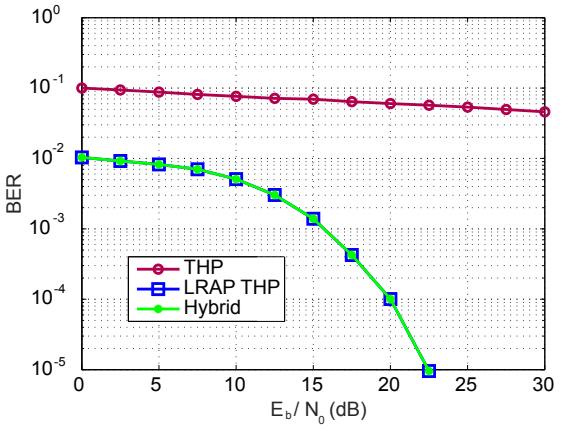

(b) Hybrid precoding with combination of THP (40\%) and LRAP THP (60\%)

Figure 6: BER performance for a $4 \times 4$ MIMO satellite channel with deep shadow and elevation angle $10^{\circ}(\alpha=-24.4 \mathrm{~dB}, \psi=9.4 \mathrm{~dB}, M P=-23.9 \mathrm{~dB}$ and $K=0 \mathrm{~dB})$.

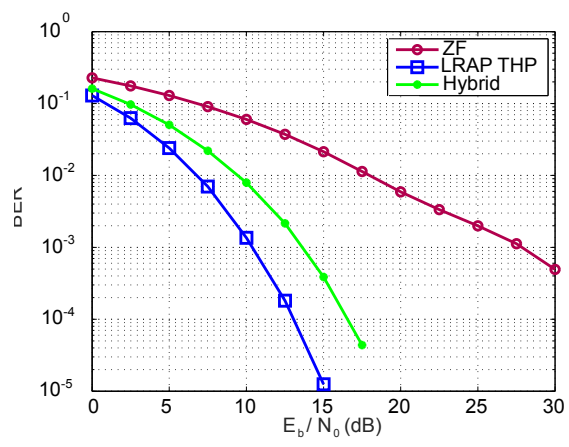

(a) Hybrid precoding with combination of ZF (70\%) and LRAP THP (30\%)

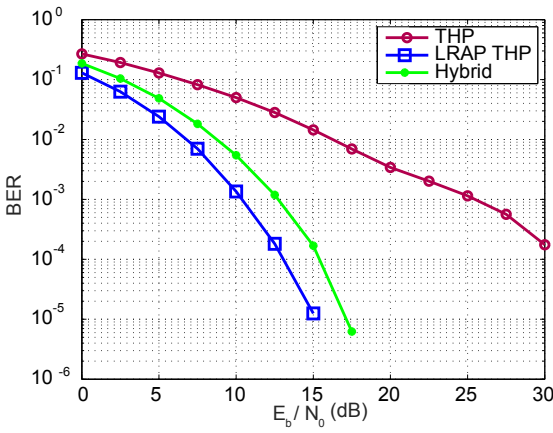

(b) Hybrid precoding with combination of THP (70\%) and LRAP THP (30\%)

Figure 7: BER performance for a $4 \times 4$ MIMO satellite channel with line-of-sight and elevation angle $40^{\circ}(\alpha=-0.2 \mathrm{~dB}, \psi=1 \mathrm{~dB}, M P=-32.9 \mathrm{~dB}$ and $K=0 \mathrm{~dB})$.

It is proved that the combined method can achieve the LRAP THP performance with much less complexity. 


\section{CONCLUSIONS}

An analysis of the precoding performance for the MIMO satellite channel performance has been carried out. Satellite channels exhibit a better behavior under LOS conditions. As the shadow increases, communication performance gets worse. Nonetheless, the use of precoders can mitigate this issue, and good values of BER can be obtained by using LRAP.

Notwithstanding, a computational cost study of the different precoding algorithms was carried out. It was shown that the LRAP have higher cost than the $\mathrm{ZF}$ and the THP ones. This fact limits the use of these precoders because even though they exhibit the best performance, their costs can be potentially too high when the system's size increases. For this reason, it is important to reach a compromise between the desired BER and the computational cost. A good solution in order to achieve the required performance at low computational cost, is a hybrid scheme. This scheme is able to adapt to a bad environment based on the condition number of the channel matrix in order to reach successfully the requirements desired.

This way, computational cost on the transmitter could be reduced without losing performance. These benefits are especially noteworthy when the $L_{c h}$ is low and the deep shadow increases.

\section{ACKNOWLEDGMENTS}

This work has been partially funded by the Spanish MINECO Grant RACHEL TEC2013-47141-C4-4-R and through FPU AP-2012/71274.

\section{REFERENCES}

[1] S. Yang, L. Hanzo, Fifty years of MIMO detection: The road to large-scale MIMO, IEEE Communications Surveys \& Tutorials 17 (4) (2015) 19411988. 
[2] J. Kyröläinen, A. Hulkkonen, J. Ylitalo, A. Byman, B. Shankar, P.-D. Arapoglou, J. Grotz, Applicability of MIMO to satellite communications, International Journal of Satellite Communications and Networking 32 (4) (2014) 343-357.

[3] G. Mabrouk, G. Hamad, Y. K. Badran, Modeling and performance evaluation of dual polarized MIMO land mobile satellite channels, International Journal of Computer Applications 96 (21) (2014) 14-20.

[4] P. D. Arapoglou, A. Ginesi, S. Cioni, S. Erl, F. Clazzer, S. Andrenacci, A. Vanelli-Coralli, DVB-S2X-enabled precoding for high throughput satellite systems, International Journal of Satellite Communications and Networking 34 (3) (2016) 439-455.

[5] V. Joroughi, M. Á. Vázquez, A. I. Pérez-Neira, Generalized multicast multibeam precoding for satellite communications, IEEE Transactions on Wireless Communications 16 (2) (2017) 952-966.

[6] M. Á. Vázquez, M. B. Shankar, C. Kourogiorgas, P.-D. Arapoglou, V. Icolari, S. Chatzinotas, A. D. Panagopoulos, A. I. Pérez-Neira, Precoding, scheduling and link adaptation in mobile interactive multibeam satellite systems, IEEE Journal on Selected Areas in Communications (2018) 1-9.

[7] M. Costa, Writing on dirty paper (corresp.), IEEE transactions on information theory 29 (3) (1983) 439-441.

[8] M. Á. Vázquez, A. Perez-Neira, D. Christopoulos, S. Chatzinotas, B. Ottersten, P.-D. Arapoglou, A. Ginesi, G. Tarocco, Precoding in multibeam satellite communications: Present and future challenges, IEEE Wireless Communications 23 (6) (2016) 88-95.

[9] S. Roger, F. Domene, A. Gonzalez, V. Almenar, G. Piñero, An evaluation of precoding techniques for multiuser communication systems, Wireless Communication Systems (ISWCS), 2010 7th International Symposium on (2010) 295-299. 
[10] J. Wang, Y. Xiao, S. Li, L. Li, J. Zhang, Performance evaluation of precoding in spatial modulation OFDM on a LTE channel, Communication Technology (ICCT), 2012 IEEE 14th International Conference on (2012) $1188-1192$.

[11] S. Roger, A. Gonzalez, V. Almenar, A. Vidal, Combined k-best sphere decoder based on the channel matrix condition number, Communications, Control and Signal Processing, 2008. ISCCSP 2008. 3rd International Symposium on (2008) 1058-1061.

[12] K. P. Liolis, J. Gómez-Vilardebó, E. Casini, Statistical Modeling of DualPolarized MIMO Land Mobile Satellite Channels, IEEE Transactions on Communications 58 (11) (2010) 3077-3083.

[13] R. Adeogun, Capacity and Error Rate Analysis of MIMO Satellite Communication Systems in Fading Scenarios, International Journal of Electrical and Computer Engineering (IJECE) 4 (4) (2014) 614-622.

[14] N. Lawrence, L. Davis, D. Haley, A polarimetric line-of-sight channel model for MIMO satellite communications, Communications Theory Workshop (AusCTW), 2013 Australian (2013) 99-104.

[15] F. P. Fontán, M. Vázquez-Castro, C. E. Cabado, J. P. García, E. Kubista, Statistical modeling of the LMS channel, IEEE Transactions on Vehicular Technology 50 (6) (2001) 1549-1567.

[16] C. Windpassinger, R. F. Fischer, T. Vencel, J. B. Huber, Precoding in multiantenna and multiuser communications, IEEE Transactions on Wireless Communications 3 (4) (2004) 1305-1316.

[17] B. M. Hochwald, C. B. Peel, A. L. Swindlehurst, A vector-perturbation technique for near-capacity multiantenna multiuser communication-part II: Perturbation, IEEE Transactions on Communications 53 (3) (2005) 537544. 
[18] C. B. Peel, B. M. Hochwald, A. L. Swindlehurst, A vector-perturbation technique for near-capacity multiantenna multiuser communication-part I: channel inversion and regularization, IEEE Transactions on Communications 53 (1) (2005) 195-202.

[19] S. Roger, A. Gonzalez, V. Almenar, A. M. Vidal, Extended LLL algorithm for efficient signal precoding in multiuser communication systems, IEEE Communications Letters 14 (3) (2010) 220-222.

[20] D. Wubben, D. Seethaler, J. Jaldén, G. Matz, Lattice reduction, IEEE Signal Processing Magazine 28 (3) (2011) 70-91.

[21] A. K. Lenstra, H. W. Lenstra, L. Lovász, Factoring polynomials with rational coefficients, Mathematische Annalen 261 (4) (1982) 515-534.

[22] C. Windpassinger, R. F. Fischer, J. B. Huber, Lattice-reduction-aided broadcast precoding, IEEE Transactions on Communications 52 (12) (2004) 2057-2060.

[23] D. Xu, Y. Huang, L. Yang, Improved nonlinear multiuser precoding using lattice reduction, Signal, image and video processing 3 (1) (2009) 47-52.

[24] G. H. Golub, C. F. Van Loan, Matrix computations, Vol. 3, JHU Press, 2012.

[25] S. Roger, A. Gonzalez, V. Almenar, A. M. Vidal, MIMO channel matrix condition number estimation and threshold selection for combined K-best sphere decoders, IEICE Transactions on Communications 92 (4) (2009) 1380-1383.

M. Ángeles Simarro is currently working as a research in the Institute of Telecommunications and Multimedia Applications at UPV, Spain. She received her Ph.D from Universitat Politècnica de València in 2017. Her research interest include MIMO detection and precoding techniques and channel coding. 
Beatriz Puig graduated Universitat Politècnica de València in 2016. She is currently working as Salesforce Solutions Assistant.

F.J. Martínez-Zaldívar received the Licenciado en Informática and Ph.D. degrees from the Universitat Politècnica de València, Spain, in 1990 and 2007 respectively. He is currently Associate Professor at the Departamento de Comunicaciones, Universitat Politècnica de València. His current research interests include parallel computing in signal processing.

Alberto Gonzalez serves as Professor at Universitat Politècnica de València (UPV). He graduated from Politecnica de Catalunya in 1991 in Telecommunication Engineering. In 1997 he was awarded a Doctorate (PhD) from UPV. Currently, he is heading the Audio and Multimedia Digital Signal Processing research group at UPV. Dean of the Telecommunications Engineering School since June 2012, having previously been head of the Communications Department. 